\title{
Worldwide survey reveals lower susceptibility of African Aedes aegypti mosquitoes to diverse strains of Zika virus
}

Fabien Aubry ${ }^{1}$, Daria Martynow ${ }^{1}$, Artem Baidaliuk ${ }^{1}$, Sarah H. Merkling ${ }^{1}$, Laura B. Dickson ${ }^{1}$, Claudia M. Romero-Vivas ${ }^{2}$, Anubis Vega-Rúa ${ }^{3}$, Isabelle Dusfour ${ }^{4}$, Davy Jiolle ${ }^{5,6}$, Christophe Paupy ${ }^{5,6}$, Martin N. Mayanja ${ }^{7}$, Julius J. Lutwama ${ }^{7}$, Alain Kohl ${ }^{8}$, Veasna Duong ${ }^{9}$, Alongkot Ponlawat ${ }^{10}$, Van-Mai CaoLormeau $^{11}$, Richard G. Jarman ${ }^{12}$, Cheikh T. Diagne ${ }^{13}$, Oumar Faye ${ }^{14}$, Ousmane Faye ${ }^{14}$, Amadou A. Sall ${ }^{14}$, Louis Lambrechts ${ }^{1 *}$

${ }^{1}$ Insect-Virus Interactions Group, Department of Genomes and Genetics, Institut Pasteur, CNRS UMR 2000, Paris, France

${ }^{2}$ Laboratorio de Enfermedades Tropicales, Departamento de Medicina, Fundación Universidad del Norte, Barranquilla, Colombia,

${ }^{3}$ Laboratory of Vector Control Research, Environment and Health Unit, Institut Pasteur de la Guadeloupe, Guadeloupe

${ }^{4}$ Vector Control and Adaptation, Institut Pasteur de la Guyane, Vectopole Amazonien Emile Abonnenc, Cayenne, French Guiana

${ }^{5}$ MIVEGEC, IRD, CNRS, Université de Montpellier, Montpellier, France

${ }^{6}$ Centre International de Recherches Médicales de Franceville, Franceville, Gabon

${ }^{7}$ Department of Arbovirology, Uganda Virus Research Institute, Entebbe, Uganda

${ }^{8}$ MRC-University of Glasgow Centre for Virus Research, Glasgow, United Kingdom

${ }^{9}$ Virology Unit, Institut Pasteur in Cambodia, Phnom Penh, Cambodia

${ }^{10}$ Department of Entomology, Armed Forces Research Institute of Medical Sciences, Bangkok, Thailand

${ }^{11}$ Institut Louis Malardé, Papeete, Tahiti, French Polynesia

${ }^{12}$ Viral Diseases Branch, Walter Reed Army Institute of Research, Silver Spring, Maryland, United States of America

${ }^{13}$ Institut Pasteur Dakar, Medical Entomology Unit, Senegal

${ }^{14}$ Institut Pasteur Dakar, Arbovirus and Viral Hemorrhagic Fevers Unit, Senegal

*Corresponding author: louis.lambrechts@pasteur.fr

\section{Abstract:}

Zika virus (ZIKV) is a flavivirus mainly transmitted to humans through the bite of infected Aedes aegypti mosquitoes. First isolated in Uganda in 1947, ZIKV was shown to circulate in enzootic sylvatic cycles in Africa and Asia for at least half a century before the first reported human epidemic occurred in 2007 on the Pacific island of Yap, Micronesia. Subsequently, larger ZIKV outbreaks were recorded in French Polynesia and other South Pacific islands during 2013-2014. In 2015, ZIKV reached Brazil from where it rapidly spread across the Americas and the Caribbean, causing hundreds of thousands of human cases. The factors that have fueled the explosiveness and magnitude of ZIKV emergence in the Pacific and the Americas are poorly understood. Reciprocally, the lack of major human epidemics of ZIKV in regions with seemingly favorable conditions, such as Africa or Asia, remains largely unexplained. To evaluate the potential contribution of vector population diversity to ZIKV epidemiological patterns, we established dose-response curves for eight field-derived $A e$. aegypti populations representing the global range of the species, following experimental exposure to six low-passage ZIKV strains spanning the current viral genetic diversity. Our results reveal that African Ae. aegypti are significantly less susceptible than non-African Ae. aegypti across all ZIKV strains tested. We suggest that low susceptibility of vector populations may have contributed to prevent large-scale human transmission of ZIKV in Africa. 
bioRxiv preprint doi: https://doi.org/10.1101/342741; this version posted June 10,2018 . The copyright holder for this preprint (which was

\section{Main text:}

Zika virus (ZIKV) is a mosquito-borne virus (genus: Flavivirus; family: Flaviviridae) mainly transmitted among humans through the bite of infected Aedes aegypti mosquitoes [1]. ZIKV was first isolated in 1947 from the serum of a sentinel rhesus monkey in the Zika Forest of Uganda [2]. Subsequently, serological evidence and virus isolation from humans and mosquitoes revealed ZIKV circulation in enzootic sylvatic cycles both in Africa (African ZIKV lineage) and Asia (Asian ZIKV lineage). For at least half a century following its discovery, ZIKV did not cause any recorded human epidemic, as less than 20 human cases were documented between 1947 and 2006 [1,3].

The first ZIKV human outbreak occurred in 2007 on the Pacific island of Yap in the Federated States of Micronesia, Oceania where ZIKV (Asian lineage) infected $73 \%$ of the islanders [4]. ZIKV outbreaks were then reported in French Polynesia [5] and other South Pacific islands during 2013-2014 [6]. Whereas human ZIKV infection is usually asymptomatic or results in a self-limiting mild illness, for the first time it was associated with severe neurological complications such as congenital microcephaly and Guillain-Barré syndrome $[7,8]$. In 2015, ZIKV reached Brazil and rapidly spread across South, Central, and North America and the Caribbean [3], causing an estimated 583,451 suspected and 223,477 confirmed cases between 2015 and 2017 [9].

To date, the factors that promoted the rapid emergence, dispersal and apparent increase in pathogenicity of ZIKV in the Pacific and the Americas are not fully understood. They may include a number of mechanisms, including viral mutations increasing transmission from humans to mosquitoes, enhancing fetal microcephaly, and modulating the host immune response [10-14]. Reciprocally, the lack of major epidemics in regions such as Africa and Asia, where conditions are seemingly favorable, is still largely unexplained. It has been hypothesized that this may reflect higher levels of background immunity conferred by cross-protective antibodies against ZIKV-related viruses [15].

Besides ZIKV evolution or contrasted human background immunity, the potential contribution of vector population genetic diversity to ZIKV epidemiological patterns may have been overlooked [15]. Earlier studies using field-derived Ae. aegypti populations demonstrated significant variation in ZIKV vector competence at different geographical scales [16-21]. However, none of these studies alone considered the entire geographical range of Ae. aegypti. Likewise, the ZIKV strains previously tested did not span the extent of ZIKV genetic diversity. Here, we established dose-response curves for eight fieldderived Ae. aegypti populations from across the global distribution of the species (Table 1) exposed to six low-passage ZIKV strains (Table 2) representing the current breadth of viral genetic diversity (Figure 1). 


\begin{tabular}{cccc}
\hline Country & Locality & Year & Generations \\
\hline Thailand & Kamphaeng Phet & 2013 & $13-14$ \\
Cambodia & Phnom Penh & 2015 & $8-9$ \\
Colombia & Barranquilla & 2017 & $2-3$ \\
French Guiana & Cayenne & 2015 & $5-6$ \\
Guadeloupe & Saint François & 2015 & $6-7$ \\
Gabon & Lopé & 2014 & $9-11$ \\
Uganda & Zika & 2016 & $4-5$ \\
Cameroon & Bénoué & 2014 & $7-8$ \\
\hline
\end{tabular}

Table 1. Aedes aegypti colonies included in this study. The country of origin, locality, year of collection and number of generations spent in the laboratory prior to this study are indicated.

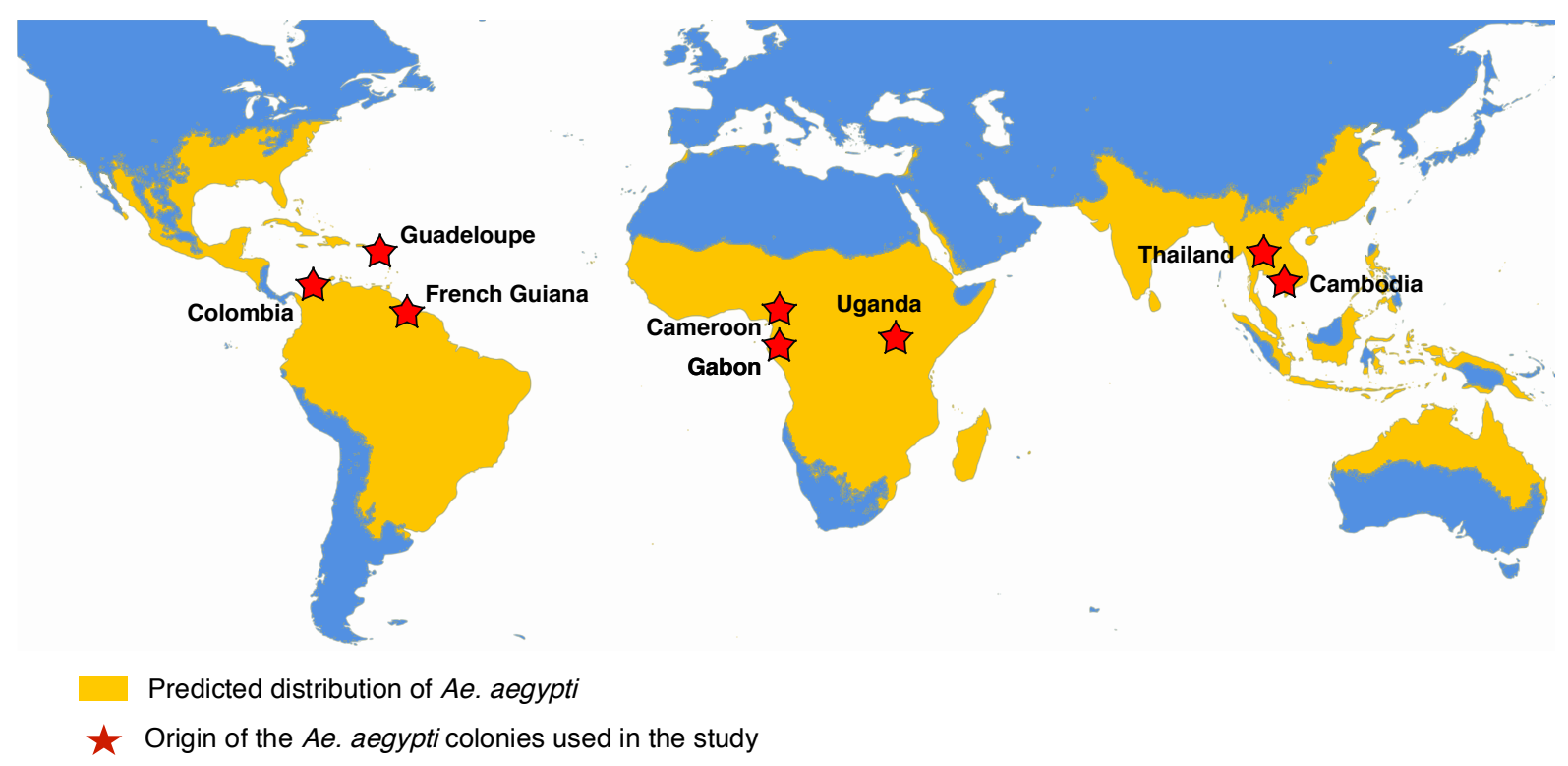

Figure 1. Geographical origin of field-derived Ae. aegypti colonies used in this study. The locations of origin of the colonies are indicated by the red stars, overlaid with the approximate global distribution of Ae. aegypti adapted from Kraemer et al. [22].

\begin{tabular}{cccc}
\hline Country & Source & Year & Passage history \\
\hline Thailand & Human serum & 2014 & TS-1; C6/36-2; Vero-1 \\
Cambodia & Human serum & 2010 & Vero 2; SM-1 \\
Philippines & Human serum & 2012 & TS-1; C6/36-2; Vero-1 \\
French Polynesia & Human serum & 2013 & C6/36-3 \\
Puerto Rico & Human serum & 2015 & Vero-2; C6/36-2 \\
Senegal & Pool of Aedes spp. and Mansonia spp. 2015 & C6/36-1 \\
\hline
\end{tabular}

Table 2. ZIKV strains included in this study. The country of origin, source of viral isolation, year of collection and passage history prior to the study are indicated. TS: Toxorhynchites splendens mosquitoes; C6/36: Aedes albopictus cells; Vero: green monkey kidney cells; SM: suckling mouse brains. 
bioRxiv preprint doi: https://doi.org/10.1101/342741; this version posted June 10,2018. The copyright holder for this preprint (which was not certified by peer review) is the author/funder, who has granted bioRxiv a license to display the preprint in perpetuity. It is made available under aCC-BY-NC-ND 4.0 International license.

Field-derived Ae. aegypti colonies from Africa (Cameroon, Uganda, Gabon), America (Colombia, Guadeloupe, French Guiana) and Asia (Thailand, Cambodia) were simultaneously challenged by oral exposure to one of six ZIKV strains, including one strain of the African lineage (Senegal 2015), three pre-epidemic (Philippines 2012 , Cambodia 2010, Thailand 2014) and two epidemic (French Polynesia 2013, Puerto Rico 2015) strains of the Asian lineage. The artificial infectious blood meal consisted of a mixture of washed erythrocytes and viral stock (three different concentrations) produced in $\mathrm{C} 6 / 36$ cells, supplemented with ATP. Upon exposure, blood engorged mosquitoes were maintained at $28^{\circ} \pm 1^{\circ} \mathrm{C}$ under $12 \mathrm{~h}$ dark:12h light cycle and $70 \%$ relative humidity. After 7 days of incubation, whole mosquito bodies were processed for RNA extraction and RT-PCR to estimate ZIKV infection prevalence.

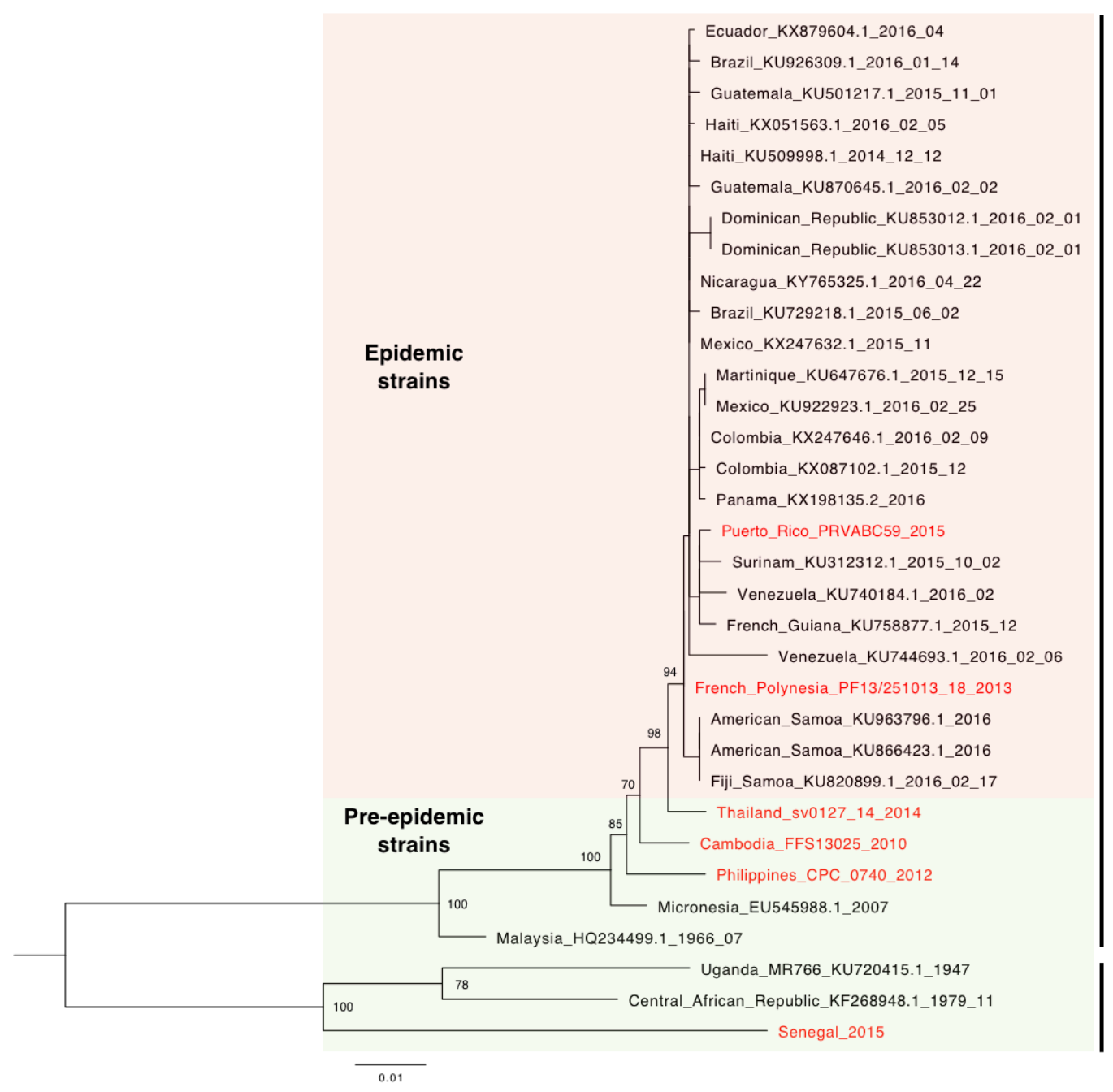

Asian

lineage

African

lineage

Figure 2. Phylogenetic position of ZIKV strains used in this study. Maximum-likelihood tree based on ZIKV envelope gene sequences shows ZIKV strains used in this study (in red font) among a background set of ZIKV strains (in black font) spanning the current viral genetic diversity. The light red background represents 'epidemic' ZIKV strains isolated during a human outbreak. The light green background represents 'pre-epidemic' ZIKV strains isolated in non-epidemic context, with the exception of the Micronesia strain, which caused a human outbreak in 2007. Bootstrap support values are shown at the relevant nodes and the scale bar represents the number of substitutions/sites. 
A total of 3,113 female Ae. aegypti were individually scored for ZIKV infection and the overall prevalence was $52.7 \%$. Multivariate logistic regression showed that the infection status depended on a three-way interaction between infectious dose, virus strain and mosquito population ( $p=0.0238$ ), indicating that the dose-response curves differed significantly among virus-population pairs (Figure 3).

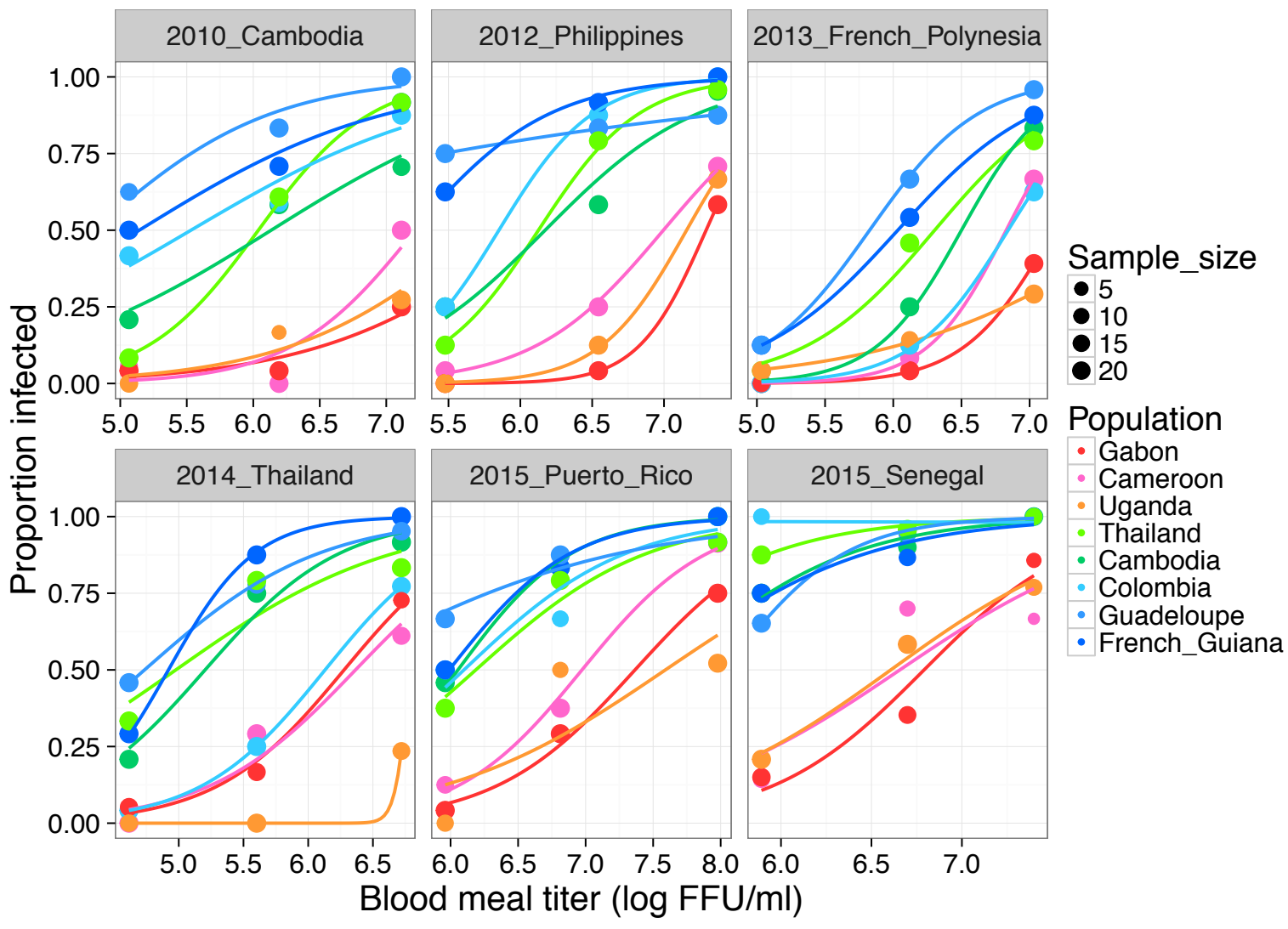

Figure 3. Dose-response curves of eight field-derived Ae. aegypti colonies challenged by six lowpassage ZIKV strains. The percentage of ZIKV-infected mosquitoes 7 days post oral challenge is shown as a function of the blood meal titer. Each panel represents a different ZIKV strain. Lines are logistic regressions of the data, color-coded by mosquito population. FFU: focus-forming units.

When mosquito populations were nested within their continent of origin (Asia, Africa, Americas) in the statistical model, there was a strong effect of the continent ( $p<$ 0.0001), which was mainly driven by the significantly lower susceptibility of the three African mosquito populations. This was reflected in their $50 \%$ oral infectious dose $\left(O D_{50}\right)$, defined as the blood meal titer resulting in $50 \%$ prevalence. Across ZIKV strains, the OID $_{50}$ estimates obtained from the dose-responses curves ranged from 6.3 to $8.1 \log _{10} \mathrm{FFU} / \mathrm{ml}$ for the three African populations whereas they ranged from 4.7 to $6.8 \log _{10} \mathrm{FFU} / \mathrm{ml}$ for the five nonAfrican populations (Figure 4). 


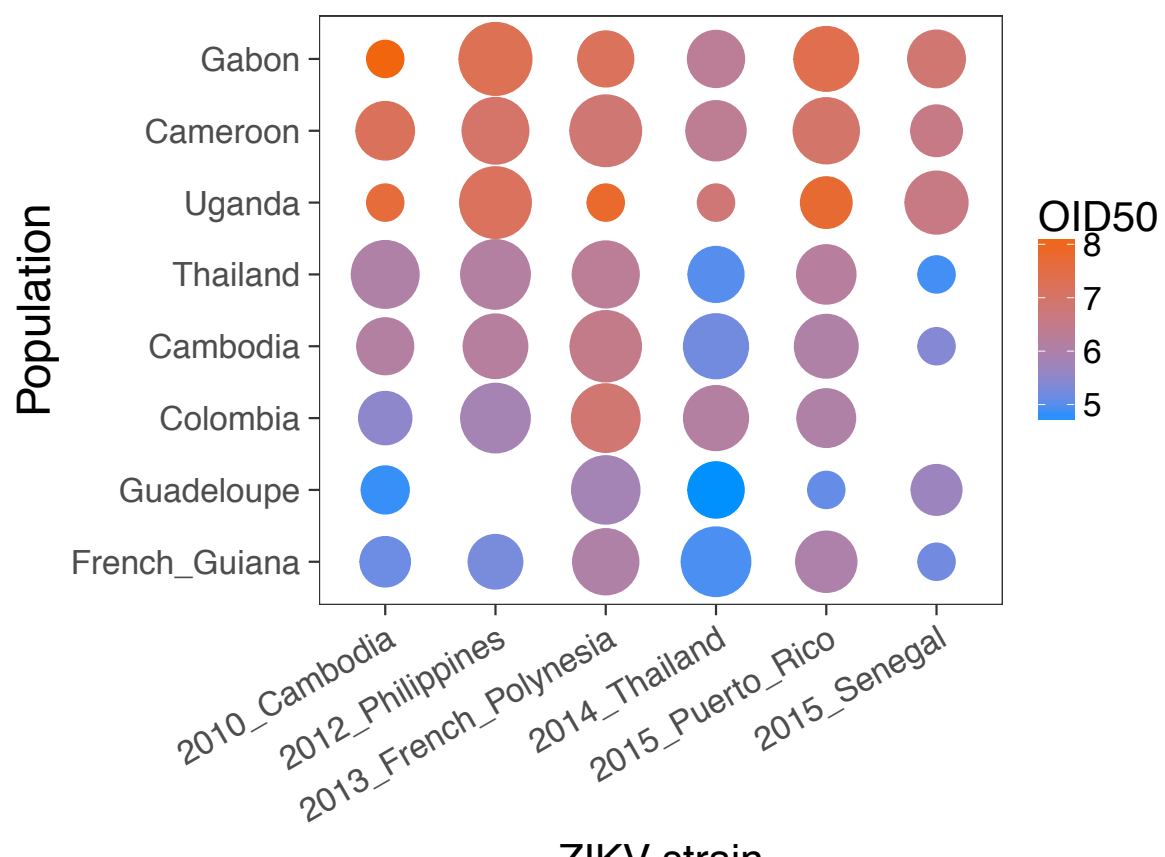

Figure 4. African Ae. aegypti are less susceptible to ZIKV infection. OID $_{50}$ estimates are shown for each virus-population pair on a color scale in $\log _{10} \mathrm{FFU} / \mathrm{ml}$. The size of the dot is inversely proportional to the size of the confidence interval of the $\mathrm{OID}_{50}$ estimate. When the size of the confidence interval could not be estimated it was arbitrarily set to $2 \log _{10}$ units. Lack of a dot means that the $\mathrm{OID}_{50}$ could not be estimated with the data.

Our data indicate that Ae. aegypti colonies of African origin are, overall, less susceptible to all ZIKV strains tested than Ae. aegypti colonies of non-African origin. Oral susceptibility does not directly translate into vector competence because the latter also depends on subsequent steps of virus dissemination and transmission. However, it is tempting to speculate that the lower susceptibility of African Ae. aegypti populations may have contributed to prevent large-scale human ZIKV outbreaks in Africa. Exceptions are Gabon where ZIKV presumably circulated in the human population in 2007 but was likely transmitted by Ae. albopictus [23] and Cabo Verde where a human ZIKV outbreak occurred in 2015 off the coast of West Africa [24]. Conversely, our data fail to provide an explanation to the lack a major human epidemic in Asia because the two Asian Ae. aegypti populations tested had similar levels of ZIKV susceptibility as American populations. The clear dichotomy observed in ZIKV susceptibility between our African and non-African Ae. aegypti colonies mirrors the two main genetic clusters of global Ae. aegypti populations [25]. Elucidating the genetic basis of this natural difference in ZIKV susceptibility could help to unravel the mechanisms of ZIKV acquisition by mosquitoes. 


\section{Acknowledgements:}

This work was primarily funded by the European Union's Horizon 2020 research and innovation programme under ZikaPLAN grant agreement no. 734584. It was partially supported by the European Union's Horizon 2020 research and innovation programme under ZIKAlliance grant agreement no. 734548.

\section{Author contributions:}

Designed and coordinated the study: FA, LL. Performed the experiments: FA, DM, AB, SHM, LBD. Contributed biological materials: CMRV, AVR, ID, DJ, CP, MNM, JJL, AK, VD, AP, VMCL, RGJ, CTD, OumF, OusF, AAS. Analyzed the data: FA, LL. Wrote the paper: FA, LL.

\section{References:}

1. Boyer S, Calvez E, Chouin-Carneiro T, Diallo D, Failloux AB. An overview of mosquito vectors of Zika virus. Microbes Infect. 2018. Epub ahead of print.

2. Dick GW, Kitchen SF, Haddow AJ. Zika virus. I. Isolations and serological specificity. Trans R Soc Trop Med Hyg. 1952;46(5):509-20.

3. Musso D, Gubler DJ. Zika Virus. Clin Microbiol Rev. 2016;29(3):487-524.

4. Duffy MR, Chen TH, Hancock WT, Powers AM, Kool JL, Lanciotti RS, et al. Zika virus outbreak on Yap Island, Federated States of Micronesia. N Engl J Med. 2009;360(24):2536-43.

5. Cao-Lormeau VM, Roche C, Teissier A, Robin E, Berry AL, Mallet HP, et al. Zika virus, French polynesia, South pacific, 2013. Emerg Infect Dis. 2014;20(6):1085-6.

6. Cao-Lormeau VM, Musso D. Emerging arboviruses in the Pacific. Lancet. 2014;384(9954):1571-2.

7. Cauchemez S, Besnard M, Bompard P, Dub T, Guillemette-Artur P, Eyrolle-Guignot D, et al. Association between Zika virus and microcephaly in French Polynesia, 2013-15: a retrospective study. Lancet. 2016;387(10033):2125-32.

8. Oehler E, Watrin L, Larre P, Leparc-Goffart I, Lastere S, Valour F, et al. Zika virus infection complicated by Guillain-Barre syndrome--case report, French Polynesia, December 2013. Euro Surveill. 2014;19(9).

9. The Pan American Health Organization (PAHO) / World Heath Organization (WHO). Zika Cumulative Cases [cited 2018 May 31]. Available from: https://www.paho.org/hq/index.php?option=com_content\&view=article\&id=12390 \&ltemid=42090\&lang=en.

10. Fritz R, Blazevic J, Taucher C, Pangerl K, Heinz FX, Stiasny K. The unique transmembrane hairpin of flavivirus fusion protein $E$ is essential for membrane fusion. J Virol. 2011;85(9):4377-85.

11. Pettersson JH, Eldholm V, Seligman SJ, Lundkvist A, Falconar AK, Gaunt MW, et al. How Did Zika Virus Emerge in the Pacific Islands and Latin America? MBio. 2016;7(5). 
12. Yuan L, Huang $X Y$, Liu $Z Y$, Zhang $F$, Zhu XL, Yu JY, et al. A single mutation in the prM protein of Zika virus contributes to fetal microcephaly. Science. 2017;358(6365):9336.

13. Liu Y, Liu J, Du S, Shan C, Nie K, Zhang R, et al. Evolutionary enhancement of Zika virus infectivity in Aedes aegypti mosquitoes. Nature. 2017;545(7655):482-6.

14. Xia H, Luo H, Shan C, Muruato AE, Nunes BTD, Medeiros DBA, et al. An evolutionary NS1 mutation enhances Zika virus evasion of host interferon induction. Nat Commun. 2018;9(1):414.

15. Pettersson JH, Bohlin J, Dupont-Rouzeyrol M, Brynildsrud OB, Alfsnes K, Cao-Lormeau VM, et al. Re-visiting the evolution, dispersal and epidemiology of Zika virus in Asia. Emerg Microbes Infect. 2018;7(1):79.

16. Chouin-Carneiro T, Vega-Rua A, Vazeille M, Yebakima A, Girod R, Goindin D, et al. Differential Susceptibilities of Aedes aegypti and Aedes albopictus from the Americas to Zika Virus. PLoS NegI Trop Dis. 2016;10(3):e0004543.

17. Diagne CT, Diallo D, Faye O, Ba Y, Faye O, Gaye A, et al. Potential of selected Senegalese Aedes spp. mosquitoes (Diptera: Culicidae) to transmit Zika virus. BMC Infect Dis. 2015;15:492.

18. Fernandes RS, Campos SS, Ferreira-de-Brito A, Miranda RM, Barbosa da Silva KA, Castro MG, et al. Culex quinquefasciatus from Rio de Janeiro Is Not Competent to Transmit the Local Zika Virus. PLoS Negl Trop Dis. 2016;10(9):e0004993.

19. Jupille H, Seixas G, Mousson L, Sousa CA, Failloux AB. Zika Virus, a New Threat for Europe? PLoS Negl Trop Dis. 2016;10(8):e0004901.

20. Roundy CM, Azar SR, Rossi SL, Huang JH, Leal G, Yun R, et al. Variation in Aedes aegypti Mosquito Competence for Zika Virus Transmission. Emerg Infect Dis. 2017;23(4):625-32.

21. Uraki R, Hastings AK, Gloria-Soria A, Powell JR, Fikrig E. Altered vector competence in an experimental mosquito-mouse transmission model of Zika infection. PLoS Negl Trop Dis. 2018;12(3):e0006350.

22. Kraemer MU, Sinka ME, Duda KA, Mylne AQ, Shearer FM, Barker CM, et al. The global distribution of the arbovirus vectors Aedes aegypti and Ae. albopictus. Elife. 2015;4:e08347.

23. Grard G, Caron M, Mombo IM, Nkoghe D, Mboui Ondo S, Jiolle D, et al. Zika virus in Gabon (Central Africa)--2007: a new threat from Aedes albopictus? PLoS Negl Trop Dis. 2014;8(2):e2681.

24. Lourenco J, de Lourdes Monteiro M, Valdez T, Monteiro Rodrigues J, Pybus O, Rodrigues Faria N. Epidemiology of the Zika Virus Outbreak in the Cabo Verde Islands, West Africa. PLoS Curr. 2018;10.

25. Gloria-Soria A, Ayala D, Bheecarry A, Calderon-Arguedas O, Chadee DD, Chiappero M, et al. Global genetic diversity of Aedes aegypti. Mol Ecol. 2016;25(21):5377-95. 